\title{
Control Difuso Versus PID para un Sistema de Bola y Plataforma
}

\section{Diffuse Versus PID Control for a Ball and Platform System}

Presentación: 17/11/2017

Aprobación: 02/12/2017

\section{José Jorge Penco}

Universidad Tecnológica Nacional - Facultad Regional Concordia - Argentina

jpenco@frcon.utn.edu.ar

\section{Mario Roberto Modesti}

Universidad Tecnológica Nacional - Facultad Regional Córdoba - Argentina

mmodesti@frc.utn.edu.ar

\section{Resumen}

En este artículo se presentan los resultados obtenidos en la performance de funcionamiento lograda mediante un controlador PID convencional y un controlador difuso, aplicados al control de una planta multivariable, no lineal e inherentemente inestable como lo es el dispositivo de bola y plataforma, utilizado como elemento para el estudio de diferentes técnicas de control. A partir del modelo matemático del sistema se diseñaron ambos controladores empleando la metodología clásica y la estructura de Mamdani respectivamente utilizando las herramientas computacionales de Matlab ${ }^{\circledast}$. El procedimiento de verificación se realizó mediante la aplicación de consignas de entrada tendientes a imprimir a la bola una trayectoria circular y una rectangular. La comparación de las respuestas obtenidas por simulaciones muestra una clara ventaja en favor del controlador diseñado con técnicas difusas, aun considerando la presencia de ruido generado en los sensores y la aparición de eventuales perturbaciones externas.

Palabras clave:controladores pid, controladores difusos, modelado, simulación, control de sistemas no lineales 


\begin{abstract}
This article presents the results obtained in the operating performance achieved by means of a conventional PID controller and a diffuse controller, applied to the control of a multivariable, non-linear and inherently unstable plant such as the ball and platform device, used as an element for the study of different control techniques. From the mathematical model of the system, both controllers were designed using the classical methodology and structure of Mamdani respectively using Matlab ${ }^{\circledR}$ computational tools. The verification procedure was carried out through the application of entry instructions to print a circular and rectangular path to the ball. The comparison of the responses obtained by simulations shows a clear advantage in favor of the controller designed with diffuse techniques, even considering the presence of noise generated in the sensors and the occurrence of possible external disturbances.
\end{abstract}

keywords: pid controllers, fuzzy controllers, modeling, simulation, control of non-linear systems

\title{
INTRODUCCIÓN
}

EL sistema de bola y plataforma es un dispositivo electromecánico actualmente utilizado en las universidades para el estudio de técnicas clásicas y avanzadas de control. Com-puesto por una plataforma plana cuyo único punto de apoyo está ubicado en su centro geométrico y sobre la cual una bola puede rodar libremente constituye un sistema completamente inestable a lazo abierto, ver Figura 1, evidenciando, además, características no lineales de funcionamiento.

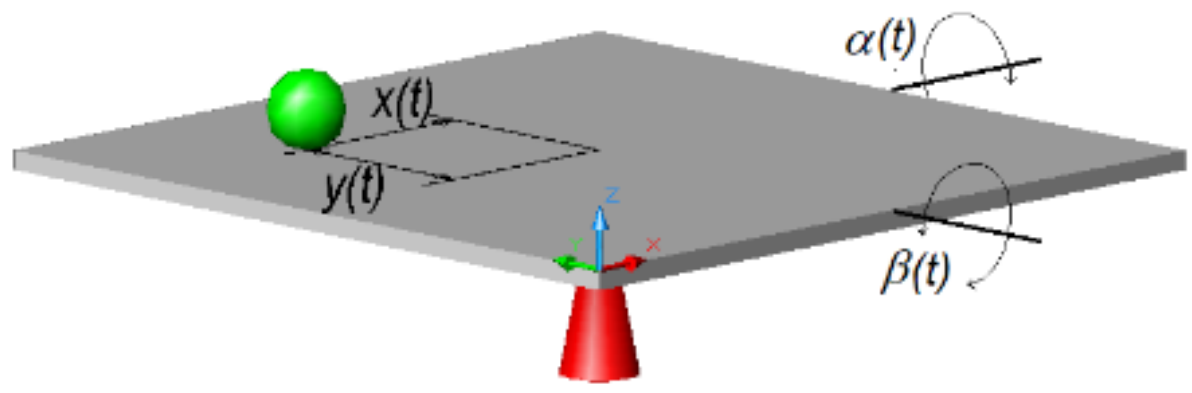

Fig. 1 Dispositivo físico de bola y plataforma

Tanto la posición estática de la bola como su desplazamiento según una determinada trayectoria pueden conseguirse manipulando adecuadamente el movimiento de la plataforma. Es necesario imprimir a esta última diferentes ángulos de inclinación para conseguir reproducir la trayectoria deseada manteniendo el equilibrio de todo el sistema. 


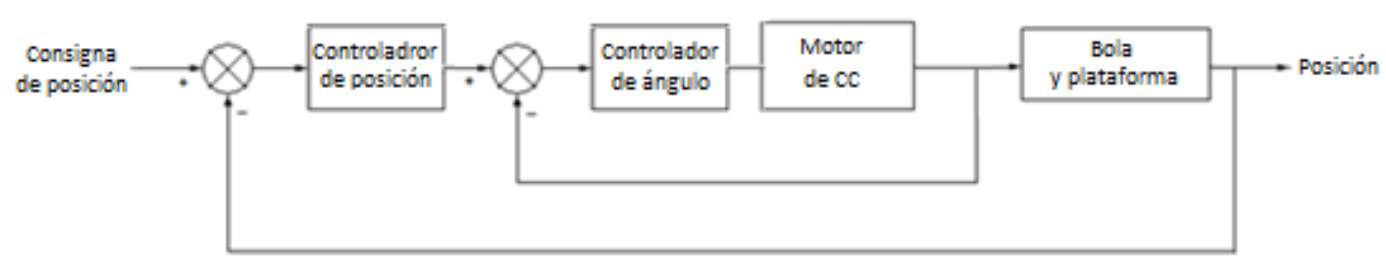

Fig. 2 Diagrama de bloques del sistema de control

Para controlar el funcionamiento de este sistema, inherentemente inestable por su naturaleza, se han desarrollado diferentes métodos como la incorporación de compensadores de adelanto de fase, diseño de reguladores cuadráticos lineales (LQR) [1] o gaussianos (LQG), diseño de observadores de estado con control realimentado, controladores PD o PID convencionales [2][3], técnicas de control difuso [4], controladores adaptivos [5] y control por modos deslizantes [6], entre otras.

En este trabajo se desarrolló la estrategia conocida como "loop in the loop" [7], Figura 2, consistente en un lazo interno funcionando como controlador de la inclinación transmitida a la plataforma, mientras que en el lazo externo se realimenta y controla finalmente la posición real de la bola sobre la placa.

Para lograr este objetivo se implementó un controlador del tipo PD en el lazo interno en tanto que para el exterior se ensayaron dos tipos de controladores diferentes, un PID de diseño clásico y el segundo, un controlador en base a reglas difusas utilizando el modelo propuesto por Mamdani.

Las simulaciones efectuadas permitieron com-parar el desempeño de ambas configuraciones y obtener conclusiones respecto a la performance de cada uno con vistas a la implementación real del sistema.

\section{MODELO MATEMÁTICO DE LA PLANTA}

Teniendo en cuenta las especificaciones de un equipo de características comerciales y utilización didáctica fueron deducidas las ecuaciones de movimiento aplicando la formulación general de Euler-Lagrange [8], y asumiendo las siguientes simplificaciones

-No hay deslizamiento de la bola sobre la plataforma

-La bola es completamente simétrica y homogénea

-Se desprecian las fricciones

-La bola se mantiene siempre apoyada en la plataforma

Para representar el desplazamiento de la bola se definieron las coordenadas $\mathrm{xb}$, yb ubicando su origen en el centro del plano y, por otro lado, la inclinación de la plataforma fue considerada mediante los ángulos a y $\beta$ respecto a los ejes x e y respectivamente, quedando en evidencia que el dispositivo contiene cuatro grados de libertad.

Por medio de consideraciones energéticas tanto cinéticas como potenciales se formularon en [8][9][10] las siguientes ecuaciones diferenciales no lineales

$$
\left(m_{b}+\frac{I_{b}}{r_{b}^{2}}\right) \ddot{x}_{b}-m_{b}\left(x_{b} \dot{\alpha}^{2}+y_{b} \dot{\alpha} \dot{\beta}\right)+m_{b} g \sin \alpha=0
$$




$$
\begin{aligned}
\left(m_{b}+\frac{I_{b}}{r_{b}^{2}}\right) \ddot{y}_{b}-m_{b}\left(y_{b} \dot{\beta}^{2}+x_{b} \dot{\alpha} \dot{\beta}\right)+m_{b} g \sin \beta=0 \\
\tau_{p x}=\left(I_{p}+I_{b}+m_{b} x_{b}^{2}\right) \ddot{\alpha}+2 m_{b} x_{b} \dot{x}_{b} \dot{\alpha}+m_{b} x_{b} y_{b} \ddot{\beta}+ \\
\quad+m_{b} \dot{x}_{b} y_{b} \dot{\beta}+m_{b} x_{b} \dot{y}_{b} \dot{\beta}+m_{b} g x_{b} \cos \alpha \\
\tau_{p y}=\left(I_{p}+I_{b}+m_{b} y_{b}^{2}\right) \ddot{\beta}+2 m_{b} y_{b} \dot{y}_{b} \dot{\beta}+m_{b} y_{b} x_{b} \ddot{\alpha}+ \\
\quad+m_{b} \dot{y}_{b} x_{b} \dot{\alpha}+m_{b} y_{b} \dot{x}_{b} \dot{\alpha}+m_{b} g y_{b} \cos \beta
\end{aligned}
$$

Los parámetros de las ecuaciones diferenciales planteadas se describen en la TABLA I.

TABLA I PARÁMETROS DEL SISTEMA

\begin{tabular}{c|l}
\hline Símbolo & Descripción \\
\hline$m_{b}$ & Masa de la bola \\
$r_{b}$ & Radio de la bola \\
$I_{b}$ & Momento de inercia de la bola \\
$I_{p}$ & Momento de inercia de la plataforma \\
$x_{b}$ & Posición de la bola en el eje $\mathrm{x}$ \\
$y_{b}$ & Posición de la bola en el eje y \\
$\dot{x}_{b}$ & Velocidad de la bola en el eje x \\
$\dot{y}_{b}$ & Velocidad de la bola en el eje y \\
$\tau_{p x}$ & Torque resultante sobre la plataforma en el eje x \\
$\tau_{p y l}$ & Torque resultante sobre la plataforma en el eje y \\
$\alpha$ & Ángulo aplicadoa la plataforma según eje x \\
$\beta$ & Ánguloaplicadoa la plataforma según eje y \\
$\dot{\alpha}$ & Velocidad angular de la plataforma según eje x \\
$\beta$ & Velocidad angular de la plataforma según eje y \\
$\ddot{\alpha}$ & Aceleración angular de la plataforma según eje x \\
$\beta$ & Aceleración angular de la plataforma según eje y \\
$\mathrm{g}$ & Aceleración de la gravedad
\end{tabular}

Las ecuaciones (1) y (2) describen el movimiento de la bola sobre la plataforma, indicando que la aceleración de la bola depende del ángulo y la velocidad angular de inclinación de la plataforma, en tanto que las ecuaciones (3) y (4) muestran como la inclinación de ésta es, asimismo, dinámicamente influenciada por la posición y velocidad de la bola. Este juego de ecuaciones diferenciales pone de manifiesto el comportamiento no lineal del sistema.

No obstante, la simetría que demuestran estas ecuaciones se observa la existencia de un

acoplamiento interno en el sistema ya que los términos $\dot{\alpha} y_{b} \dot{\beta}$ y $x_{b} \dot{\alpha} \dot{\beta}$ indican que la aceleración aplicada en el ángulo de inclinación de la plataforma, según uno de los ejes de movimiento, modifica no solamente la posición de la bola sino también el ángulo de inclinación de la plataforma con respecto al otro eje de movimiento.

Sin embargo, asegurando una baja velocidad y aceleración de la plataforma resulta que 
$|\dot{\alpha}| \ll 1$ y $|\dot{\beta}| \ll 1$, con lo cual es posible considerar despreciable el efecto de los términos de acoplamiento y por lo tanto dividir el sistema en dos subsistemas de igual estructura y que pueden controlarse de manera independiente.

En las expresiones (5) y (6) se representan las ecuaciones de estado para los subsistemas desacoplados.

$$
\left[\begin{array}{l}
\dot{x}_{1} \\
\dot{x}_{2} \\
\dot{x}_{3} \\
\dot{x}_{4}
\end{array}\right]=\left[\begin{array}{c}
x_{2} \\
B\left(x_{1} x_{4}^{2}-g \sin x_{3}\right) \\
x_{4} \\
0
\end{array}\right]+\left[\begin{array}{l}
0 \\
0 \\
0 \\
1
\end{array}\right]\left[u_{x}\right]
$$

$$
\left[\begin{array}{l}
\dot{x}_{5} \\
\dot{x}_{6} \\
\dot{x}_{7} \\
\dot{x}_{8}
\end{array}\right]=\left[\begin{array}{c}
x_{6} \\
B\left(x_{5} x_{8}^{2}-g \sin x_{7}\right) \\
x_{8} \\
0
\end{array}\right]+\left[\begin{array}{l}
0 \\
0 \\
0 \\
1
\end{array}\right]\left[u_{y}\right]
$$

siendo

$$
\begin{aligned}
& X=\left[x_{1}, x_{2}, x_{3}, x_{4}, x_{5}, x_{6}, x_{7}, x_{8}\right]^{T}=\left[x_{b}, \dot{x}_{b}, \alpha, \dot{\alpha}, y_{b}, \dot{y}_{b}, \beta, \dot{\beta}\right]^{T} \\
& U=\left[u_{x}, u_{y}\right]^{T}=[\alpha, \beta]^{T} \\
& Y=h(X)=\left(x_{1}, x_{5}\right)^{T}=\left(x_{b}, y_{b}\right)^{T}
\end{aligned}
$$

U es el vector de entradas formado por los ángulos de inclinación aplicados a la plataforma.

Por otra parte, limitando dichos ángulos en el orden de $\pm 5^{\circ}$ se tiene que con lo que es posible ignorar este factor no lineal de las ecuaciones, si bien aproximando hasta los $\pm 14^{\circ} \mathrm{el}$ error sólo es del 1\% y si, además,

$\dot{\alpha} \ll 1 \mathrm{y} \dot{\beta} \ll 1 \Rightarrow \dot{\alpha} \dot{\beta} \approx 0, \dot{\alpha}^{2} \approx 0, \dot{\beta}^{2} \approx 0$

entonces las expresiones (5) y (6) pueden ser linealizadas obteniéndose un modelo razonablemente simplificado y desacoplado que representa al sistema, el cual se indica en (7) y (8).

$$
\left[\begin{array}{l}
\dot{x}_{1} \\
\dot{x}_{2} \\
\dot{x}_{3} \\
\dot{x}_{4}
\end{array}\right]=\left[\begin{array}{cccc}
0 & 1 & 0 & 0 \\
0 & 0 & -B g & 0 \\
0 & 0 & 0 & 1 \\
0 & 0 & 0 & 0
\end{array}\right]\left[\begin{array}{l}
x_{1} \\
x_{2} \\
x_{3} \\
x_{4}
\end{array}\right]+\left[\begin{array}{l}
0 \\
0 \\
0 \\
1
\end{array}\right]\left[u_{x}\right]
$$




$$
\left[\begin{array}{l}
\dot{x}_{5} \\
\dot{x}_{6} \\
\dot{x}_{7} \\
\dot{x}_{8}
\end{array}\right]=\left[\begin{array}{cccc}
0 & 1 & 0 & 0 \\
0 & 0 & -B g & 0 \\
0 & 0 & 0 & 1 \\
0 & 0 & 0 & 0
\end{array}\right]\left[\begin{array}{l}
x_{5} \\
x_{6} \\
x_{7} \\
x_{8}
\end{array}\right]+\left[\begin{array}{l}
0 \\
0 \\
0 \\
1
\end{array}\right]\left[u_{y}\right]
$$

Con el modelo lineal obtenido, y aplicando los valores del dispositivo indicado en [8], se obtienen idénticas funciones de transferencia (9) para cada uno de los ejes de movimiento.

$$
G_{b p}(s)=\frac{7}{s^{2}}=\frac{1}{0.143 s^{2}}
$$

\section{DISEÑO DEL CONTROLADOR}

El accionamiento de la plataforma se efectúa según dos ejes perpendiculares mediante la aplicación del torque que produce los ángulos de inclinación correspondientes, los cuales son proporcionados por servomotores de corriente continua. Dichos motores son gobernados mediante la tensión de armadura, manteniendo el campo magnético constante. En estas condiciones su dinámica puede representarse por la función de transferencia lineal expresada en la ecuación (10) la cual ha sido obtenida a partir de los principios físicos que rigen su funcionamiento [11].

$$
G_{m}(s)=\frac{\theta(s)}{V_{a}(s)}=\frac{K_{m}}{s\left[\left(R_{a}+L_{a} s\right)(J s+b)+K_{b} K_{m}\right]}
$$

donde

$\mathrm{Ra}=$ Resistencia de armadura

$\mathrm{La}=$ Inductancia de armadura

$\mathrm{J}=$ Momento de inercia

$\mathrm{b}=$ Coeficiente de fricción

$\mathrm{Km}=$ Constante de par

$\mathrm{Kb}=$ Constante de f.e.m.

Considerando los parámetros de un motor real, proporcionados en [12] y despreciando el efecto de un polo de muy alta frecuencia se tiene la expresión aproximada a continuación:

$G_{m}(s)=\frac{\theta(s)}{V_{\text {in }}(s)}=\frac{274}{s(0.13 s+7.65)}$

Con el propósito de obtener una respuesta escalón cuyo sobrepaso no sea superior al 20\%, se ajustaron las siguientes funciones de transferencia para los controladores:

Controlador PD (posición del motor)

$$
G_{p d}(s)=0.005(1+1.5 s)
$$

Controlador PID (posición de la bola) 


$$
G_{p i d}(s)=0.75+\frac{0.1}{s}+1.8 s
$$

Asimismo, para el lazo de control externo se diseñó, en segundo término, un controlador difuso con el objeto de reemplazar al PID convencional y poder evaluar su desempeño en el sistema. Éste debe procesar el error producido entre la consigna de posición y la ubicación real de la bola, utilizando a tal efecto la estructura propuesta por Mamdani.

Para la obtención de los valores difusos de las señales de entrada, el error y su variación, se consideraron cinco funciones de membresía del tipo triangular y dos trapezoidales en los extremos del universo de discurso. En tanto que para generar la salida se utilizaron siete funciones de tipo triangular, ver Figura 3.

En general los ajustes de las funciones de pertenencia suponen la existencia de un experto que provee la información necesaria para determinar los valores del sistema. Para el caso de los controladores difusos puede no existir una base sólida de conocimientos para un caso específico, o una configuración que permita obtener la mejor respuesta. Por ello los parámetros de diseño se ajustan normalmente en base al conocimiento de la dinámica del sistema y, si bien existen numerosos algoritmos con el objetivo de optimizar la sintonía, en la práctica es habitual utilizar el método directo de prueba y error. Este ha sido el procedimiento empleado en el trabajo, procurando reducir el sobrepaso frente a una excitación en escalón y un bajo tiempo de establecimiento, con el objetivo de que sea posible reproducir las trayectorias de referencia manteniendo la estabilidad y un error aceptable.

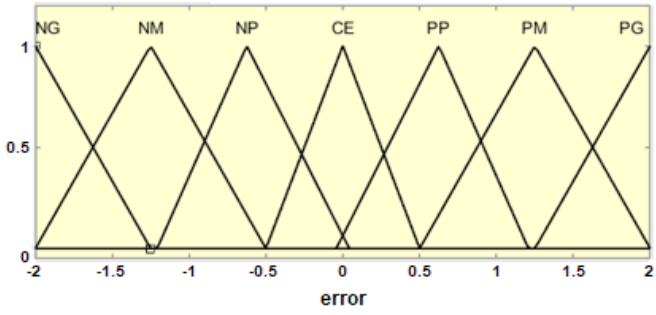

Fig. 3a Funciones de membresía para el error

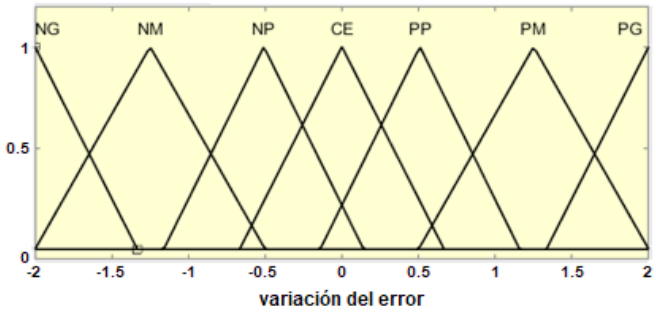

Fig. 3b Funciones de membresía para cambios del error 


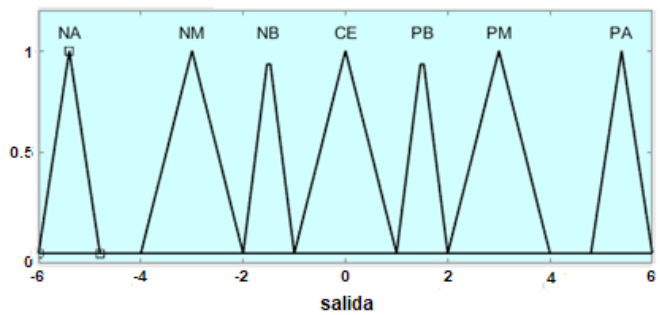

Fig. 3c Funciones de membresía para la salida

Asimismo, para optimizar la respuesta fueron calibrados los niveles de las señales de entrada y salida aplicando los ajustes de ganancia respectivos [13].

En la Figura 4 se observa el cuadro de asignación de reglas difusas programadas en el controlador, asignando a los conjuntos difusos de entrada los términos lingüísticos grande $(\mathrm{NG})$, negativo medio (NM), negativo pequeño (NP), cero (CE), positivo pequeño (PP), positivo medio $(\mathrm{PM})$ y positivo grande $(\mathrm{PG})$. En tanto que para la señal de salida se utilizaron las asignaciones negativo alto (NA), negativo medio (NM), negativo bajo (NB), cero (CE), positivo bajo (PB), positivo medio (PM) y positivo alto (PA).

\begin{tabular}{|c|c|c|c|c|c|c|c|c|}
\hline & \multicolumn{7}{|c|}{ error } \\
\hline & & $\mathrm{NG}$ & NM & NP & $\mathrm{CE}$ & PP & $\mathrm{PM}$ & PG \\
\hline \multirow{7}{*}{ 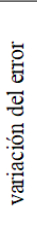 } & NG & $\mathrm{CE}$ & NB & NM & $\mathrm{NA}$ & NA & NA & NA \\
\hline & $\mathrm{NM}$ & PB & $\mathrm{CE}$ & NB & $\mathrm{NM}$ & NA & NA & NA \\
\hline & NP & $\mathrm{PM}$ & PB & $\mathrm{CE}$ & $\mathrm{NB}$ & $\mathrm{NM}$ & $\mathrm{NA}$ & NA \\
\hline & $\mathrm{CE}$ & PA & $\mathrm{PM}$ & PB & $\mathrm{CE}$ & NB & $\mathrm{NM}$ & NA \\
\hline & PP & $\mathrm{PA}$ & PA & PM & PB & $\mathrm{CE}$ & NB & $\mathrm{NM}$ \\
\hline & $\mathrm{PM}$ & PA & PA & PA & $\mathrm{PM}$ & PB & $\mathrm{CE}$ & NB \\
\hline & PG & $\mathrm{PA}$ & PA & PA & PA & $\mathrm{PM}$ & PB & $\mathrm{CE}$ \\
\hline
\end{tabular}

Fig. 4 Cuadro de asignación de reglas difusas

\section{EVALUACION DE DESEMPEÑO}

Conforme a lo expresado en las ecuaciones (5) y (6) este sistema puede ser controlado por accionamientos independientes, los cuales aplican torques en direcciones perpendiculares sobre la plataforma. Con el propósito de verificar su comportamiento se efectuaron simulaciones utilizando el software Simulink ${ }^{\circledR}$, para lo cual el sistema fue sometido a una serie de evaluaciones en las que se aplicó en primer término una consigna de trayectoria circular y posteriormente una consigna de trayectoria rectangular.

Se ensayaron diferentes niveles para la señal de entrada como también la incorporación de ruido en las señales de realimentación. Por último, se aplicaron determinadas perturbaciones sobre la plataforma para verificar la capacidad de estabilización proporcionada por el controlador.

A. Trayectoria circular

1) Controlador PID convencional: 
En primer término, se obtuvieron respuestas del sistema a partir de la utilización del controlador PID clásico. En la Figura 5 se observan los resultados para una consigna de trayectoria circular generada mediante la aplicación de señales senoidales defasadas en 90 grados. Los niveles máximos de amplitud aplicados fueron 0.25 y 0.65 respectivamente.

Asimismo, se incluyó la incorporación de ruido en las señales provenientes de los sensores. Los niveles de ruido considerados fueron 10-2 para la señal del sensor de ángulo de inclinación y 10-5 para la realimentación de posición de la bola.
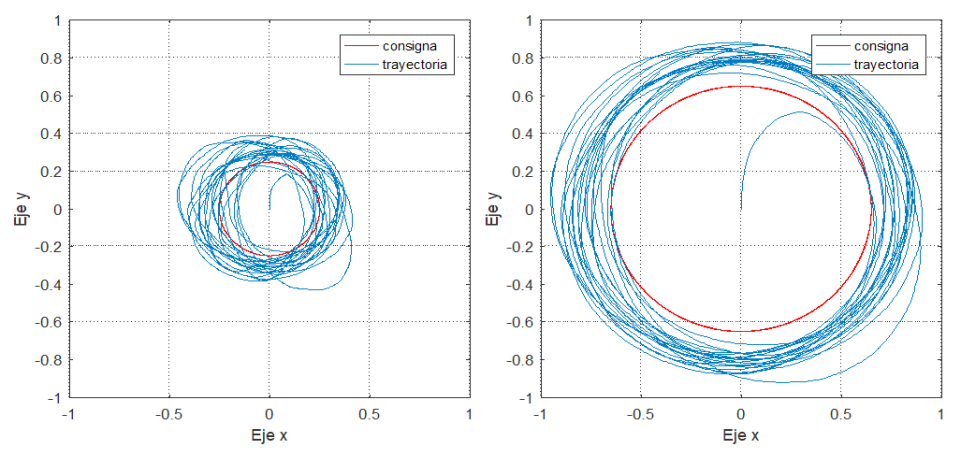

Fig. 5 Respuesta con PID convencional, diferentes niveles de señal de entrada y presencia de ruido

Las respuestas obtenidas son estables, aunque se aprecia el significativo impacto que produce la presencia de ruido, siendo en mayor medida el que afecta al lazo externo, que realimenta la posición de la bola. Por otra parte, se observa la existencia de un error de estado estacionario y cierto retardo para alcanzar el régimen permanente.

\section{2) Controlador difuso:}

Posteriormente se utilizó el controlador Mamdani, observándose en la Figura 6 las respuestas del sistema. Se introdujeron los niveles 0.25 y 0.75 destacándose que a pesar de haberse incrementado la señal de entrada la precisión resultante fue significativamente mayor. 

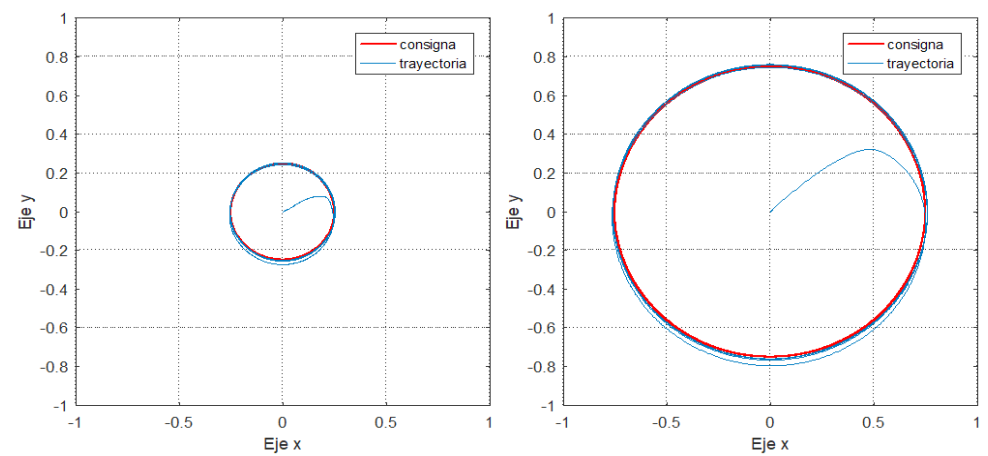

Fig. 6 Respuesta con controlador difuso y diferentes niveles de señal de entrada

En la Figura 7 se muestran las respuestas obtenidas con iguales amplitudes de señal y la incorporación del efecto producido por ruido en los sensores. Los niveles introducidos fueron 10-2 en el sensor del ángulo de inclinación de la plataforma y 10-4 para la realimentación de posición. En este caso se incrementó en diez veces el ruido incorporado en el lazo de posición, respecto a los valores referidos en la Figura 5, manteniéndose muy bajo su impacto en la trayectoria reproducida por el sistema.
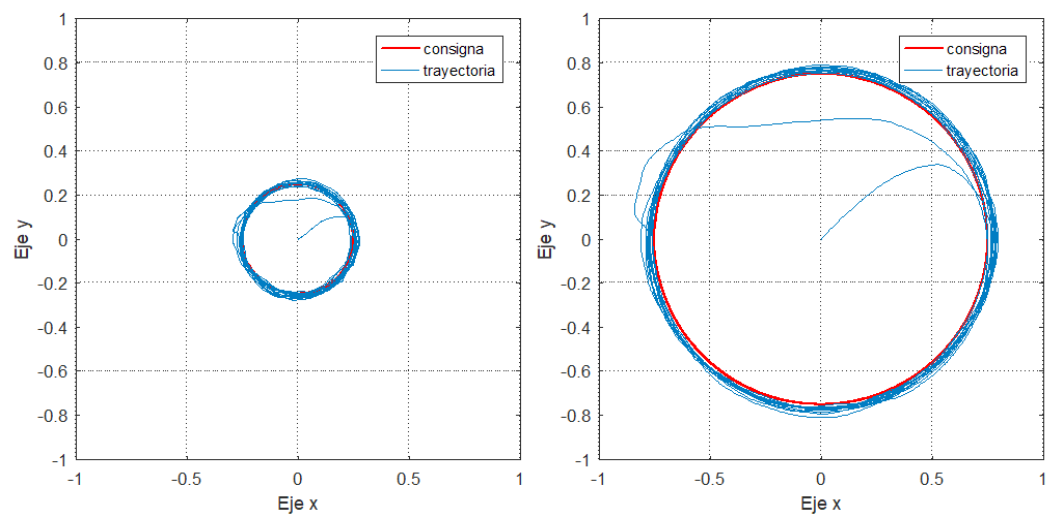

Fig. 7 Respuesta con controlador difuso, diferentes niveles de señal de entrada, con ruido y perturbaciones

En esta prueba se consideró también la ocurrencia de una breve perturbación sobre la plataforma, impactando simultáneamente sobre ambos ejes del movimiento transcurrido el 38\% del tiempo total de la simulación. La misma se aplicó con una amplitud del 50\% respecto de los valores de las señales de entrada, verificándose la capacidad de estabilización del controlador para restituir la trayectoria adecuada. En la Figura 8 se observan las señales de entrada y salida del sistema, y una representación del error entre ambas, en función del tiempo. 

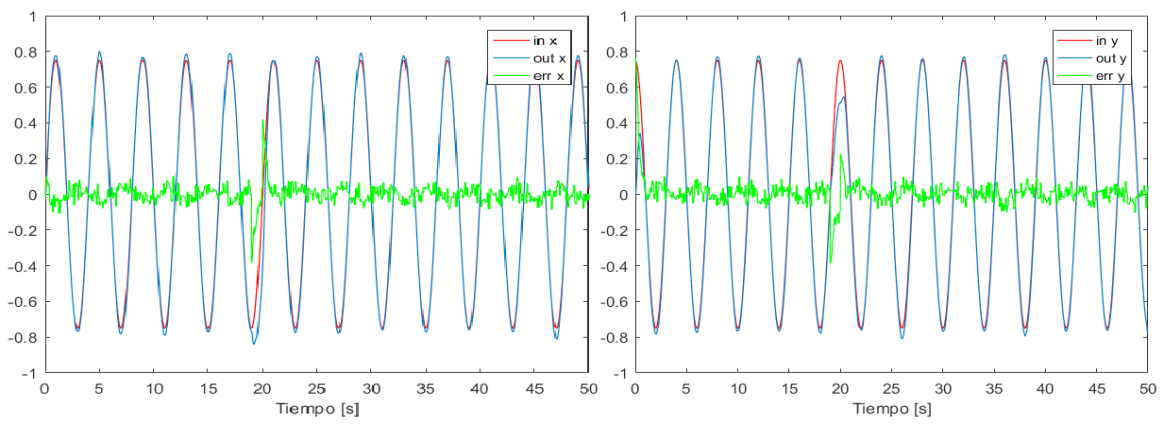

Fig. 8 Señales de entrada y salida del sistema

B. Trayectoria rectangular

1) Controlador PID convencional:

Un segundo juego de simulaciones fue realizado mediante la aplicación de una trayectoria de referencia rectangular, imponiendo una mayor exigencia para el controlador en función de los cambios bruscos de dirección.

En la Figura 9 se aprecian las trayectorias recorridas por la bola para el sistema sin afectación de ruido, y con niveles de 10-2 y 10-4 para las realimentaciones de ángulo y posición. Se observa en este último caso la pérdida de precisión que el controlador no puede recuperar.

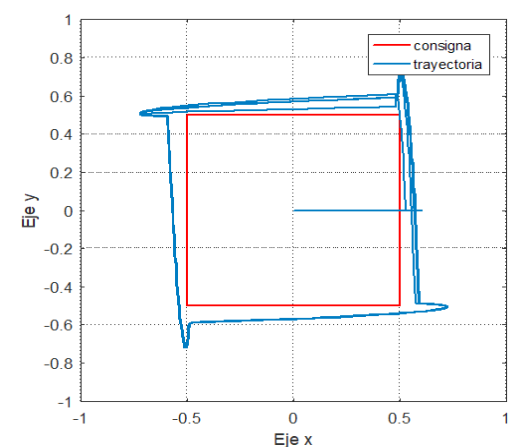

a)

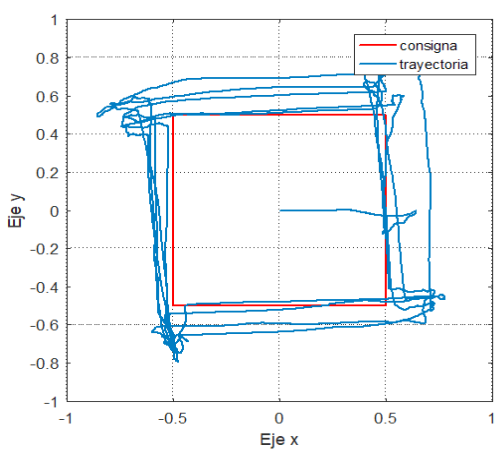

b)

Fig. 9 Respuesta con PID convencional

a) $\sin$ ruido, b) con ruido

2) Controlador difuso:

Utilizando el controlador difuso diseñado, se observa en la Figura 10 como se reproduce la trayectoria de referencia con muy baja dispersión aún con los niveles de ruido especificados 
en la prueba anterior.

Finalmente se muestran en la Figura 11 las curvas representativas de la respuesta impulsional del sistema operando con ambos tipos de controladores. Para un impulso de amplitud $100 \%$ aplicado simultáneamente en ambos ejes se obtuvo una dinámica coherente y estable, con amplitudes comparativamente admisibles entre la dimensión de la plataforma y los recorridos de la bola sobre ella.

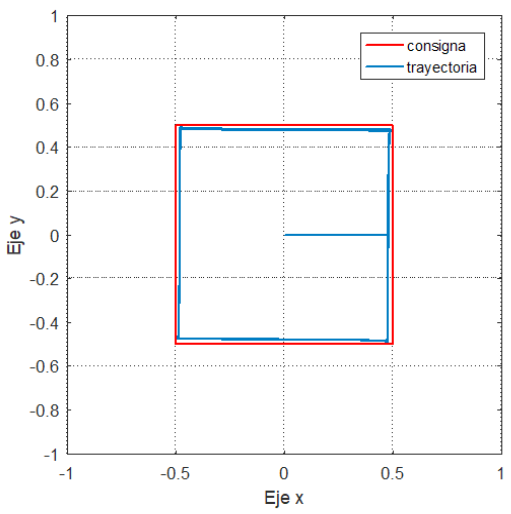

a)

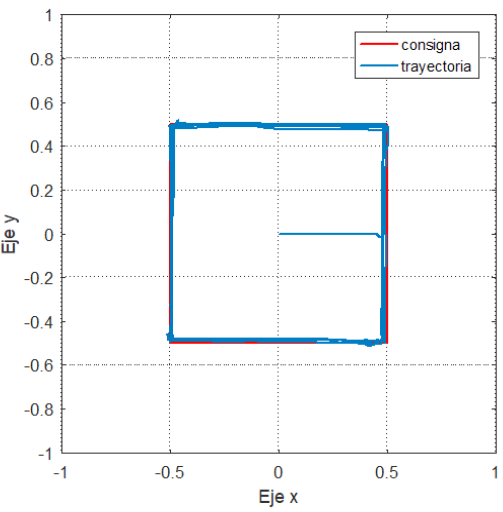

b)

Fig. 10 Respuesta con controlador difuso

a) $\sin$ ruido, b) con ruido

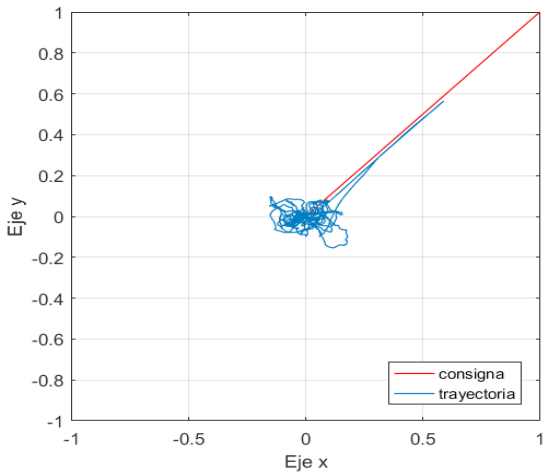

a)

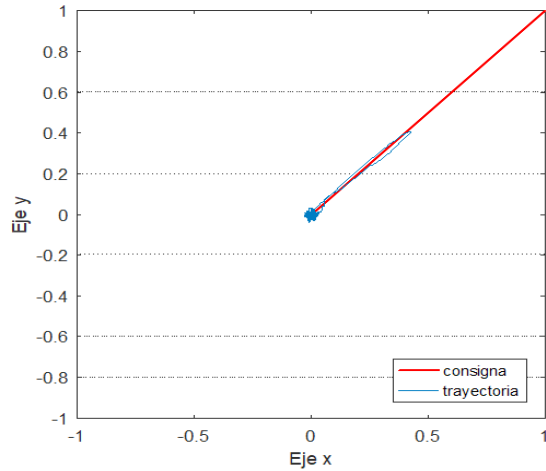

b)

Fig. 11 Respuesta impulsional del sistema con

a) PID convencional, b) controlador difuso

\section{CONCLUSIONES}


En el presente trabajo se realizó una comparación en el desempeño del sistema de control para una planta inestable mediante la utilización de un controlador PID diseñado en base a la metodología clásica y un controlador difuso basado en la estructura de Mamdani. Se evaluaron los comportamientos frente a dos trayectorias de referencia propuestas obteniéndose un comportamiento significativamente mejor del controlador difuso, anulando el error de estado estacionario y proporcionando mayor robustez frente a la perturbación con diferentes niveles de ruido en las señales de realimentación y la ocurrencia de perturbaciones desestabilizantes. Los resultados permiten interpretar que el empleo de técnicas difusas resulta ventajoso en el control de plantas o procesos que no pueden modelarse con precisión o las fuentes de información disponibles se conocen de manera inexacta o incierta.

\section{TRABAJO FUTURO}

Como trabajo futuro se plantea analizar la performance de ambos tipos de controladores sobre un modelo del sistema en cuestión que incluya los términos que aportan las características no lineales que no han sido consideradas en el presente. Asimismo, se propone ensayar una estructura del tipo ANFIS como alternativa para mejorar los resultados del control a partir de las ventajas de emplear redes neuronales artificiales adaptivas. 


\section{REFERENCIAS}

[1] M. Keshmiri, A. Jahromi, A. Mohebbi, M. Amoozgar y Wen-Fang Xie. "Modeling and Control of Ball and Beam System using Model based and Non-model based Control Approaches”. International Journal on Smart Sensing and Intelligent Systems, vol. 5, no. 1. March 2012.

[2] S. Colmenares, M. Moreno Armendáriz, C. Perez Olvera, Wen Yu, F. Ortiz Rodríguez, "Modeling and nonlinear PD regulation for ball and plate system", World Automation Congress, 2012.

[3] P. Mani Maalini, G. Prabhakar, S. Selvaperumal, "Modelling and control of ball and beam system using PID controller", International Conference on Advanced Communication Control and Computing Technologies, ICACCCT, 2016.

[4] N. Yubazaki, J. Yi, M. Otani, N. Unemura, K. Hirota, "Trajectory tracking control of unconstrained objects based on the SIRMs dynamically connected fuzzy inference model", Proceeding of 6th International Fuzzy Systems Conference, vol. 2, pp. 609-614, 1997.

[5] Y. Pattanapong, C. Deelertpaiboon, "Ball and plate position control based on fuzzy logic with adaptive integral control action", International Conference on Mechatronic and Automation, Proceeding of IEEE, Japan, August 2013.

[6] H. Liu, Y. Liang. "Trajectory tracking sliding mode control of ball and plate system”, 2nd International Asia Conference on Informatic in Control, Automation and Robotics. 2010.

[7] H. Wang, Y. Tian, Z. Sui, X. Zhang, C. Ding, "Tracking control of ball and plate system with a double feedback loop structure", Proceeding of the IEEE International Conference on Mechatronics and Automation, Harbin, China, August 2007.

[8] HUMUSOFT Ltd., CE151 Ball \& Plate Apparatus, User’s Manual, rev. 3.2, 1996-2008.

[9] X. Fan, N. Zhang, and S. Teng, "Trajectory planning and tracking of ball and plate system using hierarchical fuzzy control scheme”, Fuzzy Sets and Systems, vol. 144, pp. 297312, 2003.

[10] D. Yuan, Z. Zhang, "Modelling and control scheme of the ball-plate trajectorytracking pneumatic system with a touch screen and a rotary cylinder", Control Theory \& Applications, IET, vol. 4, no. 4, pp. 573-589, 2010.

[11] R. C. Dorf, R. H. Bishop, Modern Control Systems, 12a ed., Prentice Hall, 2011, pp 70-73.

[12] B. Messner, D. Tilbury, Control Tutorials for Matlab \& Simulink, University of Michigan and Carnegie Melon University, 2011. [Online]. Disponible en http://ctms.engin. umich.edu/

[13] K. Passino, S. Yurkovich, Fuzzy Control, Addison-Wesley Longman, Inc, 1998, pp. 78-83. 